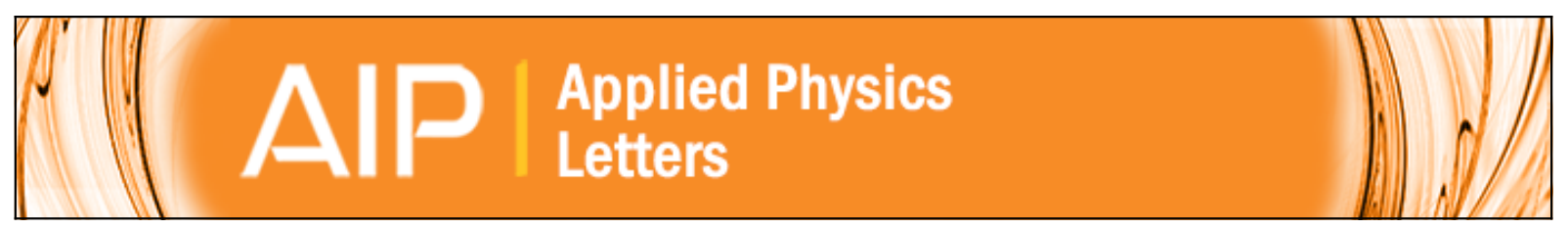

\title{
Modeling charge transfer at organic donor-acceptor semiconductor interfaces
}

Deniz Çakir, Menno Bokdam, Michel P. de Jong, Mats Fahlman, and Geert Brocks

Citation: Applied Physics Letters 100, 203302 (2012); doi: 10.1063/1.4717985

View online: http://dx.doi.org/10.1063/1.4717985

View Table of Contents: http://scitation.aip.org/content/aip/journal/apl/100/20?ver=pdfcov

Published by the AIP Publishing

\section{Articles you may be interested in}

Barrier height formation in organic blends/metal interfaces: Case of tetrathiafulvalenetetracyanoquinodimethane/Au(111)

J. Chem. Phys. 139, 214706 (2013); 10.1063/1.4836635

Charge carrier dynamics in organic semiconductors and their donor-acceptor composites: Numerical modeling of time-resolved photocurrent

J. Appl. Phys. 114, 094508 (2013); 10.1063/1.4820259

Fermi level pinning by integer charge transfer at electrode-organic semiconductor interfaces

Appl. Phys. Lett. 98, 113303 (2011); 10.1063/1.3565963

Slope parameters of the barrier heights of metal-organic contacts

Appl. Phys. Lett. 88, 112116 (2006); 10.1063/1.2178473

Organic donor/acceptor photovoltaics: The role of C 60 / metal interfaces

Appl. Phys. Lett. 82, 3101 (2003); 10.1063/1.1570936

\section{AIP $\left.\right|_{\text {APL Photonics }}$}

APL Photonics is pleased to announce Benjamin Eggleton as its Editor-in-Chief

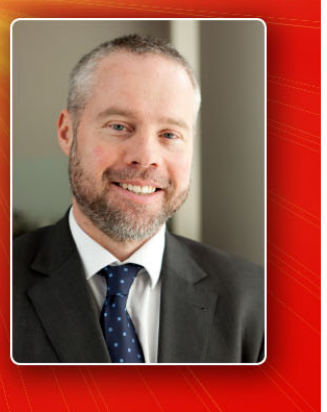




\title{
Modeling charge transfer at organic donor-acceptor semiconductor interfaces
}

\author{
Deniz Cakir, ${ }^{1}$ Menno Bokdam, ${ }^{1}$ Michel P. de Jong, ${ }^{2}$ Mats Fahlman, ${ }^{3}$ and Geert Brocks ${ }^{1, a)}$ \\ ${ }^{1}$ Computational Materials Science, Faculty of Science and Technology and MESA+Institute \\ for Nanotechnology, University of Twente, P.O. Box 217, 7500 AE Enschede, The Netherlands \\ ${ }^{2}$ MESA + Institute for Nanotechnology, University of Twente, P.O. Box 217, 7500 AE Enschede, \\ The Netherlands \\ ${ }^{3}$ Department of Physics, Chemistry and Biology, Linköping University, SE-581 83 Linköping, Sweden
}

(Received 10 January 2012; accepted 28 April 2012; published online 15 May 2012)

\begin{abstract}
We develop an integer charge transfer model for the potential steps observed at interfaces between donor and acceptor molecular semiconductors. The potential step can be expressed as the difference between the Fermi energy pinning levels of electrons on the acceptor material and holes on the donor material, as determined from metal-organic semiconductor contacts. These pinning levels can be obtained from simple density functional theory calculations. (C) 2012 American Institute of Physics. [http://dx.doi.org/10.1063/1.4717985]
\end{abstract}

Interfaces between organic materials play a crucial role in organic semiconductor devices such as light emitting diodes, field effect transistors, and organic solar cells. Band offsets at inorganic heterojunctions are determined by the chemical bonding between the two materials at the interface. Band offsets at organic heterojunctions are expected to have a different origin. Organic molecules have closed electronic shells, and interactions between such molecules are relatively weak. One expects that weak intermolecular interactions at heterojunctions do not change the electronic structure substantially. In the absence of ordered molecular dipoles, the interface dipole should then be negligible, implying that the vacuum levels left and right of the interface line up. Vacuum level line-up has indeed been observed at a number of organic-organic semiconductor heterojunctions. ${ }^{1-3}$ The small potential step of $\sim 0.1 \mathrm{eV}$ found at some organic-organic interfaces is explained by small dipoles resulting from polarization of the molecules at the interface ${ }^{4}$ and by the weak intermolecular interaction at the interface. ${ }^{5}$

A large potential step ( $\gtrsim 0.5 \mathrm{eV})$ is however observed at a number of organic-organic interfaces. It corresponds to an interface dipole originating from a significant charge displacement. The simplest explanation involves electrons transferred from donor molecules on one side of the interface to acceptor molecules on the other side. ${ }^{3}$ At some donor acceptor interfaces the transfer of electrons across the interface is demonstrated by the observation of metallic conduction along the interface. ${ }^{6,7}$

The potential step at the interface is not simply equal to the difference between the electron affinity of the acceptor molecule $A_{\mathrm{A}}$ and the ionization potential of the donor molecule $I_{\mathrm{D}}$. For example, the estimated $A_{\mathrm{A}}$ of $\mathrm{F}_{16} \mathrm{CuPc}$ is $\lesssim 4.7 \mathrm{eV}$ whereas the reported $I_{\mathrm{D}}$ of CuPc is $4.8-5.2 \mathrm{eV}^{8-10}$ Yet spontaneous electron transfer across $\mathrm{CuPc} / \mathrm{F}_{16} \mathrm{CuPc}$ interfaces is observed, ${ }^{6}$ giving rise to a potential step of $\sim 0.7 \mathrm{eV} .{ }^{10}$ It indicates that Coulomb interactions between

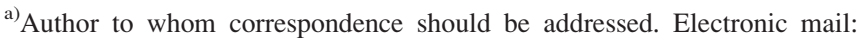
g.brocks@tnw.utwente.nl.
}

charged donor and acceptor molecules play an important role in stabilizing the charge transfer state.

Potential steps at organic-organic interfaces have been modeled by charge equilibration in a continuum density of interface states (DOIS) within the HOMO-LUMO gap, ${ }^{11}$ similar to models used for inorganic semiconductor heterojunctions. ${ }^{12}$ However, the band widths in organic semiconductors are small, ${ }^{13}$ and organic molecules have closed electronic shells, making it difficult to see why there should be a significant DOIS within the gap.

In this paper we develop a simple integer charge transfer model for the potential step at organic donor-acceptor semiconductor interfaces. ${ }^{2,3} \mathrm{We}$ focus on a single interface and do not consider phenomena introduced by additional layers or a metal electrode. The model does not involve interface states, but it includes the Coulomb interactions between charged donor and acceptor molecules.

The key result is given by Eq. (5) and the physical parameters are illustrated in Fig. 1. In a previous paper we have obtained simple expressions for the electron and hole pinning levels of organic semiconductors adsorbed on metallic substrates. ${ }^{14}$ The model developed in the current paper shows that the potential step at organic donor-acceptor semiconductor interfaces can be obtained by lining up the Fermi energy pinning level for electrons on the acceptor material to that for holes on the donor material, as illustrated in Fig. 1.

Consider an interface between a material consisting of acceptor molecules (A) and one consisting of donor molecules (D). Suppose that of the out of the $N_{\mathrm{D}}$ donor molecules at the interface, $N_{1}$ have transferred an electron to an acceptor molecule. The total energy of the interface is expressed as

$$
\begin{aligned}
E\left(N_{1}\right)= & \left(N_{\mathrm{D}}-N_{1}\right) E_{\mathrm{D}}^{0}+\left(N_{\mathrm{A}}-N_{1}\right) E_{\mathrm{A}}^{0} \\
& +N_{1}\left(E_{\mathrm{A}}^{-}+E_{\mathrm{D}}^{+}\right)+E_{\mathrm{C}}\left(N_{1}\right),
\end{aligned}
$$

where $E_{\mathrm{A}}^{0 /-}$ is the total energy of a neutral/negatively charged acceptor molecule at the interface and $E_{\mathrm{D}}^{0 /+}$ is the total energy of a neutral/positively charged donor molecule. $E_{\mathrm{C}}\left(N_{1}\right)$ is the electrostatic Coulomb energy of the interfacial 


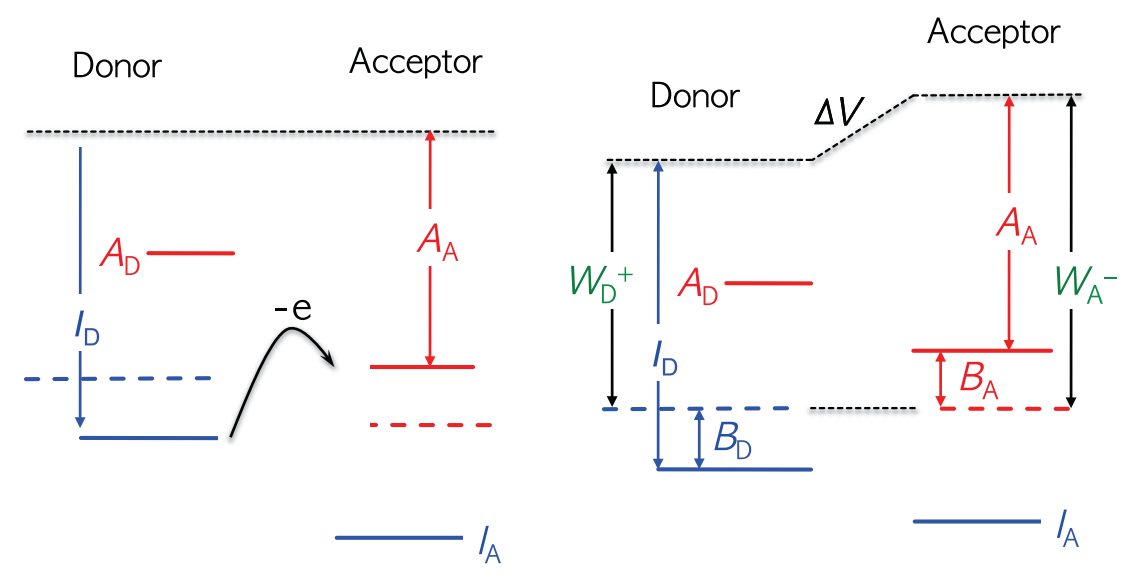

FIG. 1. Left: at an organic donor-acceptor semiconductor interface electrons are transferred. Right: at equilibrium the potential energy step $\Delta V$ is given by the difference $W_{\mathrm{A}}^{-}-W_{\mathrm{D}}^{+}$between the pinning levels for electrons on the acceptor and holes on the donor molecules.

arrangement of all charged molecules, polarization effects included. $N_{\mathrm{A}}$ is the number of acceptor molecules at the interface.

The transfer of $N_{1}$ electrons from donor to acceptor molecules gives rise to a potential energy step $\Delta V\left(N_{1}\right)$. If one measures the effect of this potential step sufficiently far from the interface, as one does in work function measurements, the molecular details of the charge distribution are not important. One can write the potential energy step in terms of an interface dipole density

$$
\Delta V\left(N_{1}\right)=\frac{e d N_{1}}{\varepsilon_{0} a_{\mathrm{A}} N_{\mathrm{A}}}=\frac{N_{1} e^{2}}{C} .
$$

Here $d$ is a dipole associated with the charge transfer between a donor and an acceptor molecule, $a_{\mathrm{A}}$ is the surface area per acceptor molecule, and $C=e \varepsilon_{0} a_{\mathrm{A}} N_{\mathrm{A}} / d$ defines the interface capacitance.

This suggests a simple model of the Coulomb energy of a donor-acceptor interface

$$
E_{\mathrm{C}}\left(N_{1}\right)=\frac{N_{1}^{2} e^{2}}{2 C}-N_{1} B_{\mathrm{D}}-N_{1} B_{\mathrm{A}} .
$$

The first term on the right-hand side is the Coulomb energy associated with charging a parallel plate capacitor. The second and third terms are the Coulomb energies associated with charging individual donor and acceptor molecules. These have to be subtracted if $E_{\mathrm{C}}$ is to represent the Coulomb interaction between the charged molecules. The charging energies of the individual molecules have been accounted for in the total energies of the molecular ions (see Eq. (1)), so they have to be subtracted in Eq. (3) to avoid double counting.

We use Eq. (3) in Eq. (1) and minimize the total energy

$$
\frac{d E\left(N_{1}\right)}{d N_{1}}=I_{\mathrm{D}}-A_{\mathrm{A}}+\frac{d E_{\mathrm{C}}\left(N_{1}\right)}{d N_{1}}=0,
$$

with $I_{\mathrm{D}}=E_{\mathrm{D}}^{+}-E_{\mathrm{D}}^{0}$ the ionization potential of a donor molecule at the interface and $A_{\mathrm{A}}=E_{\mathrm{A}}^{0}-E_{\mathrm{A}}^{-}$the electron affinity of an acceptor molecule. These molecular parameters depend on the environment as static and induced multipoles on the surrounding molecules affect the energy levels of a donor or acceptor molecule.
One can now write the potential energy step at the donor-acceptor interface, Eq. (2), as

$$
\Delta V=\left(A_{\mathrm{A}}+B_{\mathrm{A}}\right)-\left(I_{\mathrm{D}}-B_{\mathrm{D}}\right)=W_{\mathrm{A}}^{-}-W_{\mathrm{D}}^{+} .
$$

This is the main result of the model. The quantities $W^{-/+}$ correspond to the Fermi energy pinning levels for electrons/holes respectively as defined in Ref. 14. Fermi level pinning is observed at interfaces between organic materials and low (high) work function metal electrodes. It is explained in terms of electron (hole) transfer from the metal to the organic material at the interface, resulting in a work function $W^{-}\left(W^{+}\right)$of the complete system that is independent of the work function of the metal. The simple relation between the potential step at an organicorganic interface, and the work function pinning levels at metal-organic interfaces has been demonstrated experimentally for tetrathiofulvalene (TTF)/tetracyanoquinodimethan (TCNQ) interfaces. ${ }^{15}$ Obviously Eq. (5) is only valid if $W_{\mathrm{A}}^{-} \geq W_{\mathrm{D}}^{+}$. If $W_{\mathrm{A}}^{-}<W_{\mathrm{D}}^{+}$, there is no charge transfer and $\Delta V=0$.

In equilibrium the electro-chemical potential has the same value throughout the whole system, implying $\mu_{\mathrm{D}}+\Delta V\left(N_{1}\right)=\mu_{\mathrm{A}}$. Here $\mu_{\mathrm{D}}$ and $\mu_{\mathrm{A}}$ are the electrochemical potential (with respect to the local vacuum level) at the donor and acceptor side of the interface. Comparison to Eq. (5) gives $\mu_{\mathrm{A}}=W_{\mathrm{A}}^{-}, \mu_{\mathrm{D}}=W_{\mathrm{D}}^{+}$and

$$
B_{\mathrm{D}}=I_{\mathrm{D}}-\mu_{\mathrm{D}} ; \quad B_{\mathrm{A}}=\mu_{\mathrm{A}}-A_{\mathrm{A}} .
$$

These relations provide a means of extracting $B_{\mathrm{D} / \mathrm{A}}$ from experiment as the difference between the electro-chemical potential (the Fermi energy) and the ionization potential $I$ or the electron affinity $A$.

$I$ and $A$ obviously depend on the surroundings of a molecule. A highly polarizable environment stabilizes the charged state of a molecule. Changing the environment changes the polarization energy by $\Delta P$, resulting in $I \rightarrow I-\Delta P, A \rightarrow A$ $+\Delta P$. The same change in polarization also affects the molecular charging energies, i.e., $B_{\mathrm{D} / \mathrm{A}} \rightarrow B_{\mathrm{D} / \mathrm{A}}-\Delta P$. From Eq. (5) one then observes that neither the pinning levels $W^{-1+}$ nor the potential step $\Delta V$ depends on the polarization of the environment. These parameters can be obtained from the individual molecular layers. 
The same argument cannot be made for the effects resulting from the static charge distribution of the environment of a molecule. The fields from the multipoles of the molecules surrounding a donor or acceptor molecule affect the energy levels of the latter. For instance, the energy levels depend on the orientation of the molecule with respect to the surrounding molecules. Changing the packing and orientation of molecules can easily change the molecular energy levels by $\gtrsim 0.5 \mathrm{eV} .^{10,16}$

One can obtain the pinning level $W_{\mathrm{D}}^{+}$from first principles by calculating $I_{\mathrm{D}}$ and $B_{\mathrm{D}}$ for a donor molecule embedded in a molecular donor layer. Likewise $W_{\mathrm{A}}^{-}$can be obtained by calculating $A_{\mathrm{A}}$ and $B_{\mathrm{A}}$ for an acceptor molecule embedded in a molecular acceptor layer. It is shown in Ref. 14 that for calculations based on density functional theory (DFT) simplifying approximations are possible, provided one uses functionals giving total energies that are analytical in the occupation numbers of the Kohn-Sham energy levels. Commonly used functionals based on the generalized gradient approximation (GGA) or the local density approximation (LDA) have this property. The pinning levels can then be approximated by ${ }^{14}$

$$
W_{\mathrm{A}}^{-} \approx-\epsilon_{\mathrm{A}}^{\mathrm{LUMO}}+E_{\mathrm{A}, \mathrm{rel}}^{-} ; \quad W_{\mathrm{D}}^{+} \approx-\epsilon_{\mathrm{D}}^{\mathrm{HOMO}}-E_{\mathrm{D}, \mathrm{rel}}^{+},
$$

where $\epsilon_{\mathrm{A} / \mathrm{D}}$ are the Kohn-Sham LUMO/HOMO eigenvalues of the neutral acceptor/donor molecules. $E_{\mathrm{D} / \mathrm{A} \text {,rel }}^{+/-}$are the energies associated with structural relaxation upon charging the molecules with an electron or hole. These relaxation energies are relatively small $(\lesssim 0.1 \mathrm{eV})$ for the molecules considered here.

One should note that the pinning levels $W^{-1+}$ differ from the molecular A/I levels due to the electrostatic interactions of the charged molecules at the interface. In our model these differences are represented by $B_{\mathrm{A} / \mathrm{D}}$ (see Eq. (5)). The latter depends on the molecule and its environment; a typical number is $\sim 0.5 \mathrm{eV} .{ }^{13}$ It is well-known that the Kohn-Sham eigenvalues $\epsilon_{\mathrm{A} / \mathrm{D}}$ of common DFT functionals do not represent the A/I levels very well and that those functionals overestimate electron delocalization, which can lead to a spurious partial electron transfer in calculations on donoracceptor pairs. ${ }^{17}$ However, here we use a model that imposes charge transfer at an interface as an integer number of electrons. The Kohn-Sham eigenvalues then present an acceptable approximation to the pinning levels $W^{-1+}$ of the individual molecular donor and acceptor layers. ${ }^{14}$

We calculate Kohn-Sham energy levels using the Vienna $A b$ initio Simulation Package (VASP) with projector augmented waves and the PW91 GGA functional. ${ }^{18-20} \mathrm{Cal}-$ culations are performed for well-ordered molecular layers. The packing of the molecules is taken from experimental structures of molecular crystals and monolayers adsorbed on clean substrates. The unit cell in the direction perpendicular to the molecular layer is chosen sufficiently large such that the potential in the middle of the cell represents the vacuum level. The Kohn-Sham energy levels are then calculated with respect to this vacuum level.

Calculated pinning levels for different organic materials are given in Table I. The label $s$ (standing) refers to the (001) plane of the $\beta$-structure of $\mathrm{CuPc},{ }^{21}$ a similar structure for
TABLE I. Calculated pinning levels $W^{+}, W^{-}(\mathrm{eV})$ for donors and acceptors, respectively (Eq. (7)); $l$ and $s$ refer to lying and standing orientations, respectively.

\begin{tabular}{lcccc}
\hline \hline Donor & $\mathrm{CuPc}(l)$ & $\mathrm{CuPc}(s)$ & $\mathrm{T} 6(l)$ & $\mathrm{T} 6(s)$ \\
$W_{\mathrm{D}}^{+}$ & 5.18 & 4.41 & 4.65 & 3.75 \\
Acceptor & $\mathrm{F}_{16} \mathrm{CuPc}(l)$ & $\mathrm{F}_{16} \mathrm{CuPc}(s)$ & $\mathrm{C}_{60}$ & $\mathrm{PTCDA}$ \\
$W_{\mathrm{A}}^{-}$ & 4.27 & 5.21 & 4.44 & 4.74 \\
\hline \hline
\end{tabular}

$\mathrm{F}_{16} \mathrm{CuPc}$ or the low temperature (LT)-structure of sexithiophene (T6), ${ }^{22}$ which presents the surfaces with lowest energy for these crystals. The label $l$ (lying) refers to close-packed molecular layers with the molecular planes parallel to the layer. For PTCDA we use the structure where the molecules lie in the plane of the molecular layer, ${ }^{23}$ and for $\mathrm{C}_{60}$ we use the (111) plane of the fcc structure.

The pinning levels very much depend upon the orientation of the molecules within a layer. In particular, note that for the $l$ (lying) orientation of $\mathrm{CuPc}$ and $\mathrm{F}_{16} \mathrm{CuPc}$, $W_{\mathrm{A}}^{-}<W_{\mathrm{D}}^{+}$. It means that at a $\mathrm{CuPc} / \mathrm{F}_{16} \mathrm{CuPc}$ interface with the molecules in $l$ orientation with respect to the interface, there is no charge transfer. In contrast, for the $s$ orientation of $\mathrm{CuPc}$ and $\mathrm{F}_{16} \mathrm{CuPc}, W_{\mathrm{A}}^{-}>W_{\mathrm{D}}^{+}$, implying that electrons are transferred from $\mathrm{CuPc}$ to $\mathrm{F}_{16} \mathrm{CuPc}$ at the interface. Calculated potential steps are listed in Table II for all donoracceptor interfaces studied in this paper.

Experimentally, it is possible to control the orientation of the molecules within a layer by controlling the interaction with a substrate. A strong molecule-substrate interaction leads to molecular planes ending up parallel to the substrate, i.e., the $l$ orientation, whereas a weak interaction enables a molecular layer to expose its intrinsic lowest energy surface, i.e., the $s$ orientation. Comparing the calculated and experimental potential steps in Table II shows that the agreement is satisfactory. At interfaces where the calculations predict no charge transfer and thus no potential step, the experimental potential steps are small and may be attributed to weak intermolecular interactions at the interface, ${ }^{4,5}$ not considered in this paper. At the interfaces where the experimental potential steps are large, our model based on electron transfer between donor and acceptor molecules predicts the right sizes for the interfacial potential steps.

TABLE II. Calculated potential energy steps $\Delta V$ at donor/acceptor interfaces (eV), Eq. (5), compared to experimental results.

\begin{tabular}{lccccc}
\hline \hline \multicolumn{1}{c}{$\Delta V$} & Calc. & Expt. & $\Delta V$ & Calc. & Expt. \\
\hline $\begin{array}{l}\mathrm{CuPc} / \mathrm{F}_{16} \mathrm{CuPc} \\
(s)\end{array}$ & 0.80 & $0.67^{\mathrm{a}}$ & $(l)$ & 0.0 & $0.0^{\mathrm{a}}$ \\
$\begin{array}{l}\mathrm{CuPc} / \mathrm{PTCDA} \\
(s)\end{array}$ & 0.33 & $0.4^{\mathrm{b}}, 0.55^{\mathrm{c}}$ & $(l)$ & 0.0 & $0.15^{\mathrm{c}}$ \\
$\begin{array}{l}\mathrm{T} 6 / \mathrm{C}_{60} \\
(s)\end{array}$ & 0.69 & $0.6^{\mathrm{d}}$ & $(l)$ & 0.0 & $0.08^{\mathrm{e}}$ \\
\hline \hline
\end{tabular}

${ }^{\mathrm{a}}$ Reference 10 .

${ }^{\mathrm{b}}$ Reference 1 .

${ }^{\mathrm{c}}$ Reference 9 .

${ }^{\mathrm{d}}$ Reference 24

${ }^{\mathrm{e}}$ Reference 25 . 
This work is part of the European project MINOTOR (Grant No. FP7-NMP-228424). M.F. acknowledges support from STEM, the Swedish Energy Agency.

${ }^{1}$ I. Hill, D. Milliron, J. Schwartz, and A. Kahn, Appl. Surf. Sci. 166, 354 (2000).

${ }^{2}$ M. Fahlman, A. Crispin, X. Crispin, S. K. M. Henze, M. P. de Jong, W. Osikowicz, C. Tengstedt, and W. R. Salaneck, J. Phys.: Condens. Matter 19, 183202 (2007).

${ }^{3}$ S. Braun, W. R. Salaneck, and M. Fahlman, Adv. Mater. 21, 1450 (2009).

${ }^{4}$ S. Verlaak, D. Beljonne, D. Cheyns, C. R. M. Linares, F. Castet, J. Cornil, and P. Heremans, Adv. Funct. Mater. 19, 3809 (2009).

${ }^{5}$ M. Bokdam, P. A. Khomyakov, G. Brocks, Z. Zhong, and P. J. Kelly, Nano Lett. 11, 4631 (2011).

${ }^{6}$ J. Wang, H. Wang, X. Yan, H. Huang, and D. Yan, Appl. Phys. Lett. 87, 093507 (2005).

${ }^{7}$ H. Alves, A. S. Molinari, H. Xie, and A. F. Morpurgo, Nat. Mater. 7, 574 (2008).

${ }^{8}$ H. Peisert, M. Knupfer, and J. Fink, Appl. Phys. Lett. 81, 2400 (2002).

${ }^{9}$ W. Chen, D. C. Qi, Y. L. Huang, H. Huang, Y. Z. Wang, S. Chen, X. Y. Gao, and A. T. S. Wee, J. Phys. Chem. C 113, 12832 (2009).

${ }^{10}$ W. Chen, D.-C. Qi, H. Huang, X. Gao, and A. T. S. Wee, Adv. Funct. Mater. 21, 410 (2010).
${ }^{11}$ H. Vázquez, W. Gao, F. Flores, and A. Kahn, Phys. Rev. B 71, 041306(R) (2005).

${ }^{12}$ J. Tersoff, Phys. Rev. B 30, 4874(R) (1984).

${ }^{13}$ G. Giovannetti, G. Brocks, and J. van den Brink, Phys. Rev. B 77, 035133 (2008).

${ }^{14}$ M. Bokdam, D. Çakir, and G. Brocks, Appl. Phys. Lett. 98, 113303 (2011).

${ }^{15}$ S. Braun, X. Liu, W. R. Salaneck, and M. Fahlman, Org. Electron. 11, 212 (2010).

${ }^{16}$ S. Duhm, G. Heimel, I. Salzmann, H. Glowatzki, R. L. Johnson, A. Vollmer, J. Rabe, and N. Koch, Nat. Mater. 7, 326 (2008).

${ }^{17}$ G. Sini, J. S. Sears, and J.-L. Brédas, J. Chem. Theory Comput. 7, 602 (2011).

${ }^{18}$ G. Kresse and J. Hafner, Phys. Rev. B 47, (R)558 (1993).

${ }^{19}$ G. Kresse and J. Furthmüller, Phys. Rev. B 54, 11169 (1996).

${ }^{20}$ G. Kresse and D. Joubert, Phys. Rev. B 59, 1758 (1999).

${ }^{21}$ C. J. Brown, J. Chem. Soc. A 1968, 2488.

${ }^{22}$ G. Horowitz, B. Bachet, A. Yassar, P. Lang, F. Demanze, J.-L. Fave, and F. Garnier, Chem. Mater. 7, 1337 (1995).

${ }^{23}$ P. C. Rusu, G. Giovannetti, C. Weijtens, R. Coehoorn, and G. Brocks, Phys. Rev. B 81, 125403 (2010).

${ }^{24}$ Y. Ge and J. E. Whitten, Chem. Phys. Lett. 448, 65 (2007).

${ }^{25}$ R. Wang, H. Y. Mao, H. Huang, D. C. Qi, and W. Chen, J. Appl. Phys. 109, 084307 (2011). 